\title{
O químico em Portugal. Parte 1: Caracterização do profissional registado na rede social de negócios Linkedln
}

\author{
Maria Jorge Pratas*1, J. Sérgio Seixas de Melo ${ }^{1,2}$ \\ ${ }^{1}$ Sociedade Portuguesa de Química, Av. Da República 45, 3E, 1050-187 Lisboa, Portugal, sede@spq.pt \\ ${ }^{2}$ Departamento de Química, Universidade de Coimbra, 3049-535 Coimbra
}

\begin{abstract}
Portuguese Chemist. Part 1: Characterization of the professional registered on the business social network LinkedIn - The Portuguese Chemical Society launched in 2016 a web survey (A Química em Portugal, 2016, May $2^{\text {nd }}$, retrieved from http://www.spq.pt/news/540) aiming at the characterization of what can be considered nowadays a Portuguese Chemist. The main goal of the work is to equate the need of the Portuguese market for a professional chemist (no matter the degree, $B S c, M S c, P h D$ ) and if the global number of these professionals is sufficient for the needs of the country. Moreover, an additional goal was to correlate the academic background with the professional career of the respondents. Simultaneously, an employment oriented business social network, such as LinkedIn, widely used by professionals with college background, was used to get information that can be compared with the online survey results. Here we publish a first general evaluation of the LinkedIn search. A follow on article will focus on the online survey and the relevant comparisons between the two independent studies.
\end{abstract}

A sociedade Portuguesa de Química lançou em 2016 um inquérito (A Química em Portugal, 2 de maio de 2016; localizado em http://www.spq.pt/news/540) que visa a caracterização do Químico Português. O principal objetivo é perceber o enquadramento de um químico (independentemente do grau alcançado) no mercado português e se o número de licenciados formados serve as necessidades do país. Por outro lado, um dos objetivos adicionais do inquérito é correlacionar a formação académica com a situação profissional ao longo da vida. Paralelamente, utilizou-se uma rede social de negócios orientada para a empregabilidade, o LinkedIn, popular entre os quadros superiores, para comparar a informação aí encontrada com a obtida por meio do inquérito. Neste artigo apresenta-se uma primeira apreciação geral da pesquisa no LinkedIn. Seguir-se-á um segundo artigo onde serão divulgados os resultados do inquérito web e as comparações relevantes entre os dois estudos.

\section{Introdução}

Em 2008 a Sociedade Portuguesa de Química (SPQ) efetuou um estudo que visou caracterizar os Químicos em Portugal no período 1967-2007 a partir de fontes estatísticas [1]. No seguimento desse trabalho, a SPQ verificou que era necessário efetuar um inquérito direcionado, recolhido através de um sítio na rede [2], visando correlacionar os dados da formação académica com a real ocupação profissional dos inquiridos. Uma imagem similar à do inquérito pode ser obtida a partir de uma rede social de negócios cuja missão esteja fortemente orientada para o emprego e que simultaneamente tenha ampla utilização por parte dos profissionais da área que se quer caracterizar. O LinkedIn [3] é a rede social por excelência para esse fim e foi utilizada com vista a responder às perguntas sobre os Químicos Portugueses: Onde estudaram? Onde trabalham? O que fazem? Onde residem?

\section{Resultados e discussão}

\section{Área Científica}

A Figura 1 mostra a distribuição da amostra por área científica de formação académica.

Esta distribuição pode ser comparada com os valores do estudo realizado por Paulo Ribeiro-Claro e Lina Brito [1] referentes ao último ano do seu estudo (2007) onde eram indicados 38\% com formação em Engenharia Química, 35\% em Bioquímica e 27\% em Química. De notar que a pesquisa não teve qualquer limitação temporal, sen-

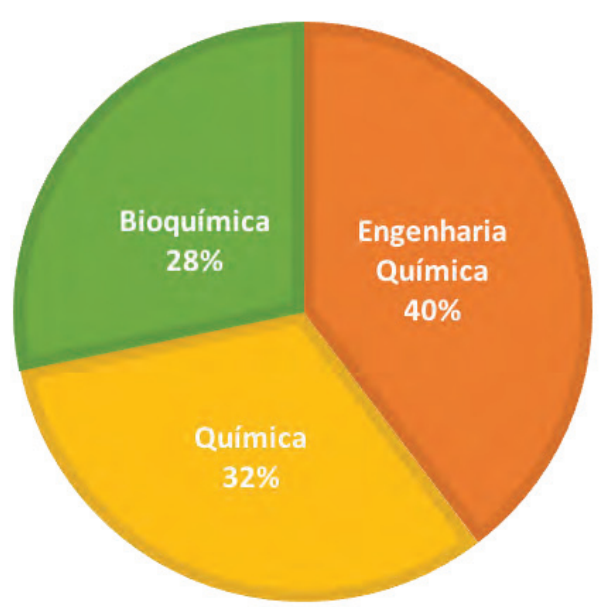

Figura 1 - Distribuição dos utilizadores por área de formação: Engenharia Química, Química e Bioquímica.

do por isso um valor integrado de todos os membros em idade ativa. Este é um dado relevante porque os cursos de Bioquímica só começaram a surgir em 1985. A prevalência da Química sobre a Bioquímica reflete ainda um pouco os dados integrados do estudo anterior em que $45 \%$ eram Engenheiros Químicos, 42\% Químicos e só 13\% Bioquímicos. Passados 10 anos desde o último estudo, e ao descartarem-se os anos mais antigos 1967-1976, verifica-se uma aproximação entre a Químicos e Bioquímicos, sendo previsível para breve uma inversão percentual. Tal reflete o número de entradas nas universidades de estudantes em Bioquímica (maioritário) relativamente aos cursos em Quí- 
mica (minoritários), em particular nos últimos 5 anos onde esta diferença se acentuou fortemente.

\section{Instituição de formação}

A Figura 2 apresenta o contributo das várias Universidades para a formação em cada uma das três grandes áreas científicas em análise. Na área da Engenharia Química a Universidade de Lisboa (através do Instituto Superior Técnico - IST) e a Universidade do Porto (pela Faculdade de Engenharia - FEUP) dominam com cerca de 2/3 do total de profissionais registados. Na área da Química continua a dominar a Universidade de Lisboa (através dos cursos ministrados na Faculdade de Ciências) com pouco menos de $1 / 3$ dos formados. Os restantes profissionais estão distribuídos de forma mais ou menos uniforme pelas Universidades Nova de Lisboa, Aveiro, Coimbra, Porto e Minho. Para a área da Bioquímica, dado ter sido introduzida nas várias Universidades de forma quase paralela, não existe uma separação tão acentuada entre as grandes Universidades (embora Lisboa, Coimbra e Porto representem 55\% dos profissionais registados), apresentando a Universidade de Coimbra uma ligeira vantagem de $(\sim 2 \%)$.

\section{Empregadores}

Dada a atomização do mercado de trabalho, esta componente do estudo limitou-se aos 25 maiores empregadores de cada área científica ficando por isso as pequenas e algumas médias empresas fora do estudo por falta de dimensão estatística. Os resultados validados (1190, dos quais 42\% em Engenharia Química e 29\% para cada uma das restan- tes áreas) apresentam-se na Figura 3. As fatias dos gráficos que se encontram ligeiramente desviadas representam universidades ou centros de investigação (Engenharia Química) e empresas (Química e Bioquímica).

A maioria dos utilizadores formados em Engenharia Química é absorvida por empresas e indústrias, ainda que 35\% sejam absorvidos por universidades e institutos de investigação. Os utilizadores formados em Química são maioritariamente absorvidos por universidades e institutos de investigação sendo a indústria farmacêutica a única a absorver uma fração significativa de profissionais. Apenas $20 \%$ dos utilizadores trabalham em empresas e indústrias. Na Bioquímica acentua-se a tendência observada na área da Química onde apenas 7\% dos utilizadores trabalham diretamente em empresas. Ressalva-se que as indústrias da área da Bioquímica são muitas vezes de pequena dimensão (em termos de número de colaboradores) e podem ter sido excluídas pelo modelo de triagem usado.

\section{Área funcional}

Apesar da limitação na resposta à questão anterior (“onde trabalham?”), os resultados obtidos na questão “o que fazem?” dá robustez a essa resposta, como se apresenta na Figura 4 com as funções desempenhadas. As fatias dos gráficos da Figura 4 que se encontram ligeiramente desviadas, representam funções ligadas à investigação e educação para todas as áreas de formação.

Dos 2550 profissionais formados em Engenharia Química cerca de $30 \%$ trabalham diretamente em Engenharia e Operações e 24\% em Investigação e Ensino. Relativa-

\section{Eng. Química}

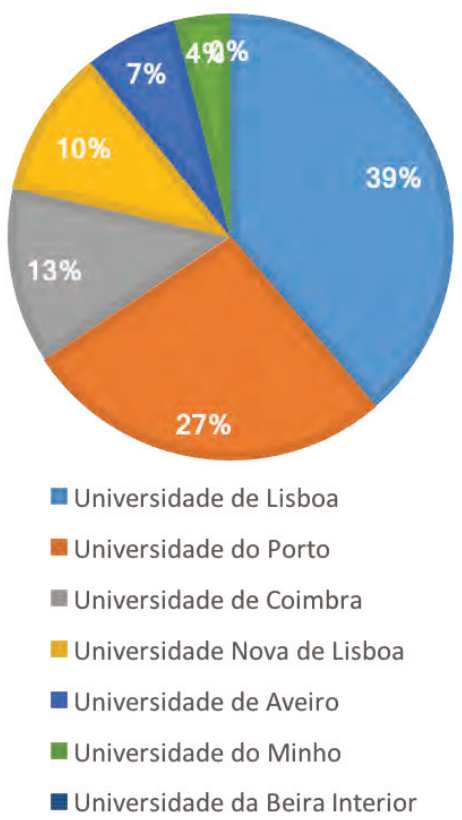

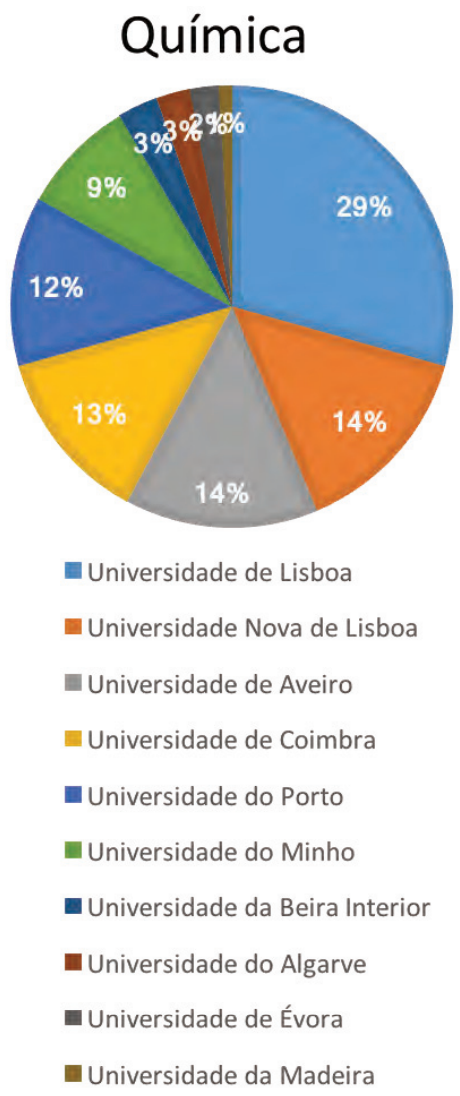

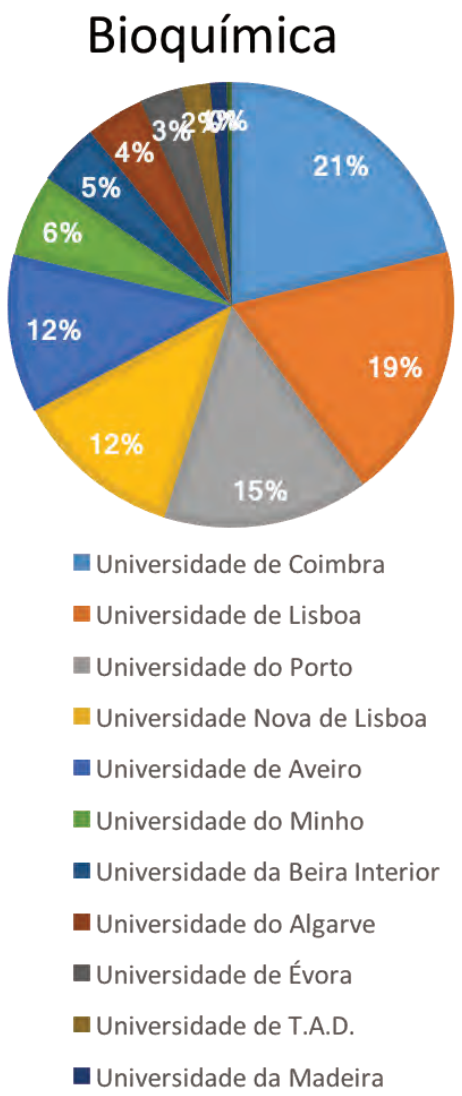

Figura 2 - Distribuição dos utilizadores do LinkdIn formados em Engenharia Química, Química e Bioquímica por instituição de ensino (Universidade de T.A.D., Universidade de Trás-os-Montes e Alto Douro). 
Eng. Química

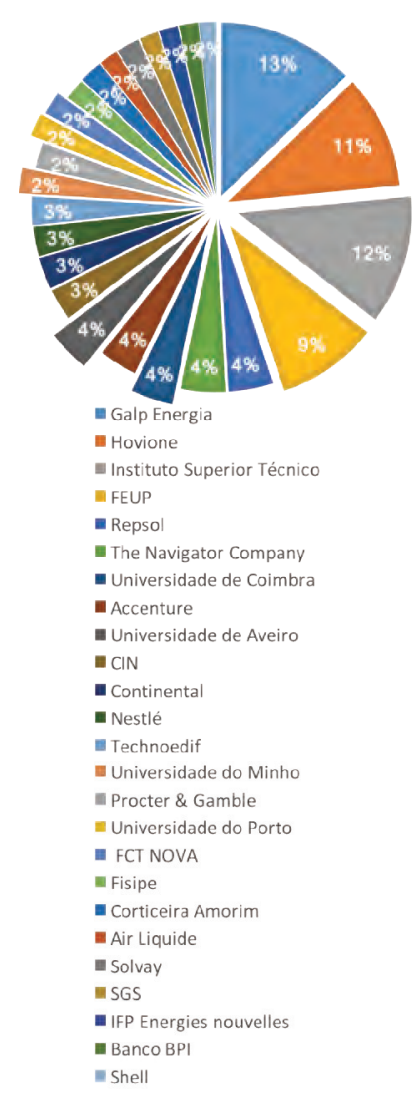

Química

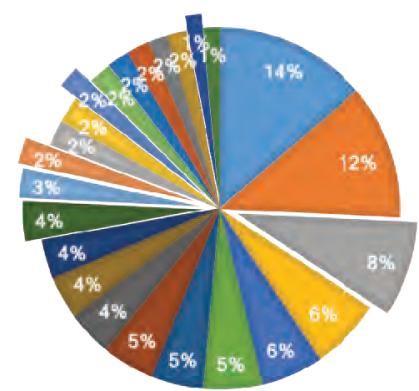

- Instituto Superior Técnico

- Universidade de Aveiro

m Hovione

- FCT NOVA

- Universidade do Minho

- Universidade de Coimbra

- CICECO - Aveiro Institute of Materials

- Universidade do Porto

- REQUIMTE- Department of Chemistry

- ITQB-UNL

- Universidade de Lisboa

- Galp

- Accenture

maurecia

- Universidade de Évora

- 3 B's Research Grous

- colep

- Universidade do Algarve

- CeNTI

- Fundação para a Ciência e Tecnologia

- Universidade Nova de Lisboa

- Instituto de Medicina Molecular

- Repsol

\section{Bioquímica}

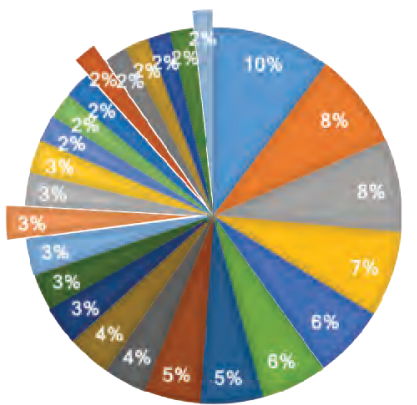

=ITQB-UNL

- Universidade de Aveiro

in Centro de Neurociências e Biologia Molecular

wnstituto de Medicina Molecular

- IBMC.INEB Laboratorio Associado

- Universidade de Coimbra

- FCT NOVA

- Instituto Gulbenkian de Ciência

- iBET - Instituto de Biologia Experimental e Tecnológica

- Life and Health Sciences Research Institute, Braga

- Universidade do Minho

- Biocant - Technology Transfer Association

- Universidade de Lisboa

- BIAL

= Universidade do Algarve

- Universidade da Beira Interior

- Universidade de Évora

- IPATIMUP

- King's College London

asK

- Instituto Superior Técnico

- MIT Portugal Program

- REQUIMTE- Department of Chemistry

- Karolinska Institutet

Hovione

Figura 3 - Distribuição dos utilizadores formados em Engenharia Química, Química e Bioquímica pelas 25 instituições mais representativas.

Eng. Química

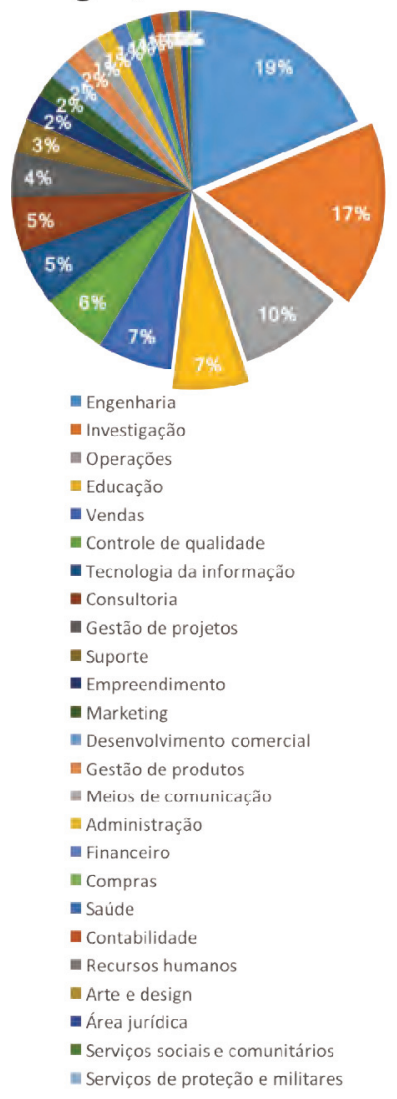

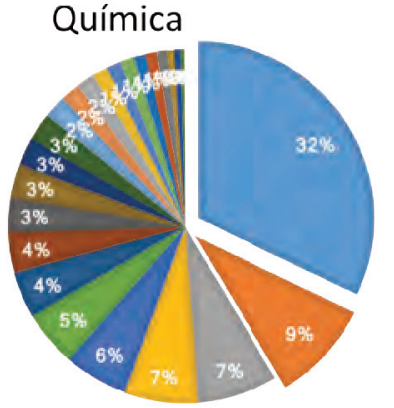

- Investigação

- Educaçăo

- Engenharia

= Operações

- Controle de qualidade

- vendas

- Tecnologia da informação

- Gestão de projetos

- Suporte

- Consultoria

- Empreendimento

- Saúde

- Meios de comunicação

- Gestão de produtos

- Desenvolvimento comercial

= Marketing

- Recursos humanos

- Área jurídica

- Financeiro

- Administração

- Arte e design

- Compras

- Contabilidade

- Serviços sociais e comunitários

- Serviços de proteção e militares

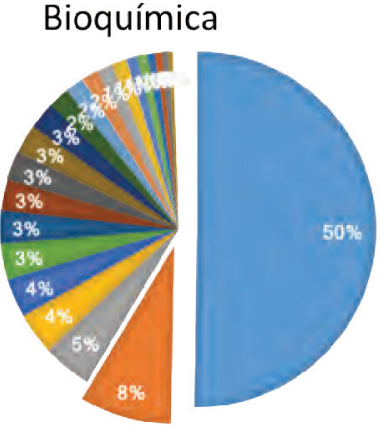

- Investigação

- Educação

- Operações

- Saúde

- Vendas

- Tecnologia da informação

- Suporte

- Engenharia

- Meios de comunicação

- Controle de qualidade

- Gestão de projetos

- Área jurídica

- Consultoria

- Administração

- Marketing

Empreendimento

- Gestão de produtos

- Desenvolvimento comercial

- Arte e design

- Contabilidade

- Serviços sociais e comunitários

- Financeiro

- Recursos humanos

- Compras

= Serviços de proteção e militares

Figura 4 - Distribuição dos utilizadores formados em Engenharia Química, Química e Bioquímica por áreas de trabalho. 
mente aos 2000 utilizadores formados em Química, cerca de $41 \%$ trabalham em Investigação e Ensino e 14\% em Engenharia e Operações. Dos 1800 profissionais de Bioquímica cerca de 50\% trabalham em Investigação e $8 \%$ no Ensino.

\section{Onde residem}

A resposta a esta questão permite perceber a distribuição destes profissionais no país e no mundo. De uma maneira geral, cerca de $70 \%$ dos utilizadores registados moram em Portugal, o que se traduz numa taxa de emigração de aproximadamente 30\% dos utilizadores formados em Química, Engenharia Química e Bioquímica, registados na plataforma LinkedIn. As regiões de Lisboa e do Porto são preferenciais para as três áreas consideradas, seguidas pela zona centro, como se apresenta na Figura 5. O destino preferencial para emigração é o Reino Unido, seguido pelos Estado Unidos da América, Europa Central e Brasil. Na Figura 6 apresentam-se os destinos preferenciais de emigração, indicados por ordem decrescente do número de profissionais formados em Química. Faz-se notar que associar o número de profissionais fora de Portugal (30\%) a uma eventual emigração forçada é pura especulação. Apesar desse efeito não poder ser negligenciado, a nossa leitura é que a rede na qual se baseia este estudo é mais usada pelos profissionais (em química) que exercem funções no estrangeiro.

\section{Conclusões}

Apesar de todas as limitações inerentes ao uso de uma rede social, tendencialmente mais usada por utilizadores

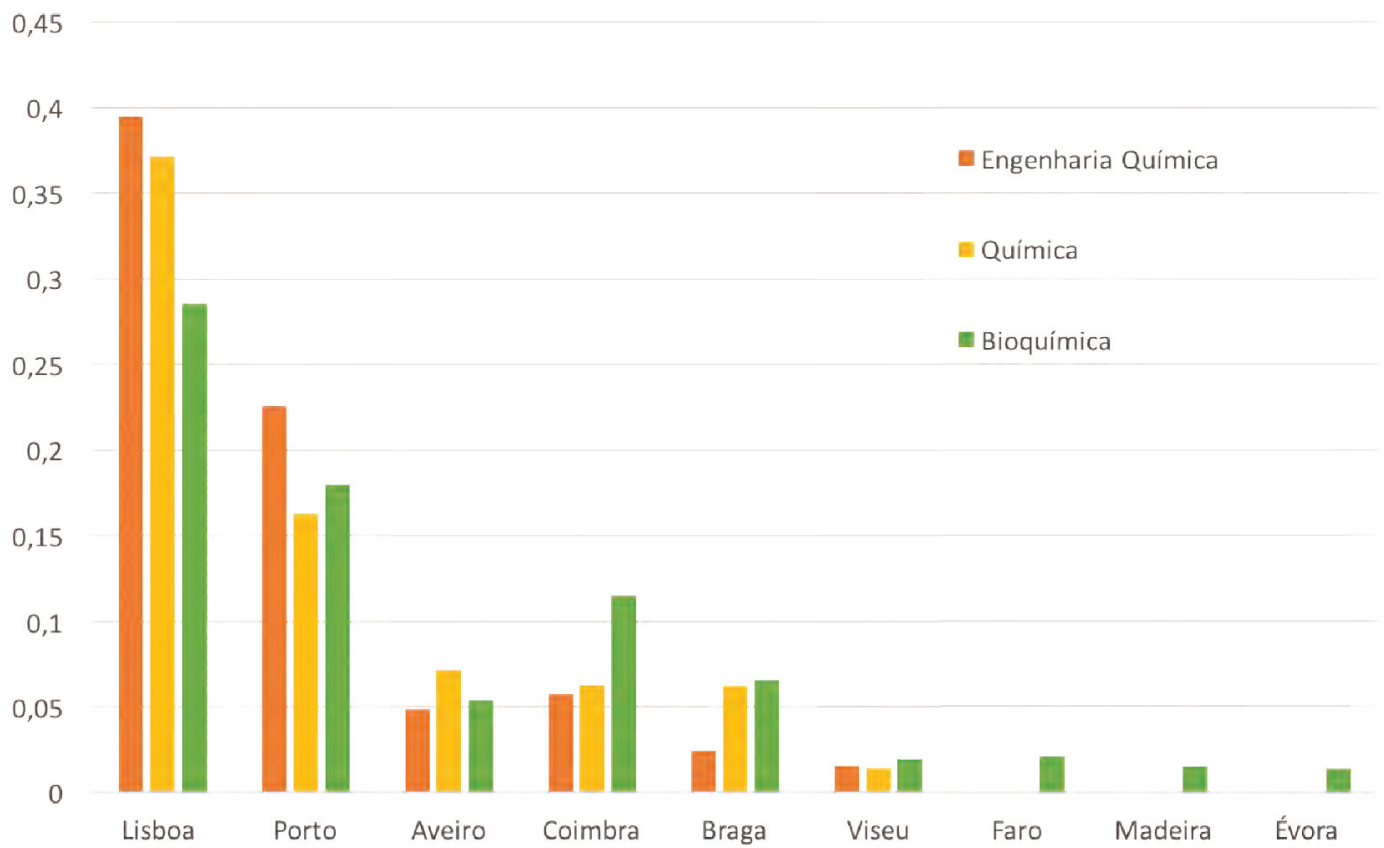

Figura 5 - Distribuição, em termos de fração, dos residentes em Portugal por região, dos utilizadores formados em Química e áreas afins.

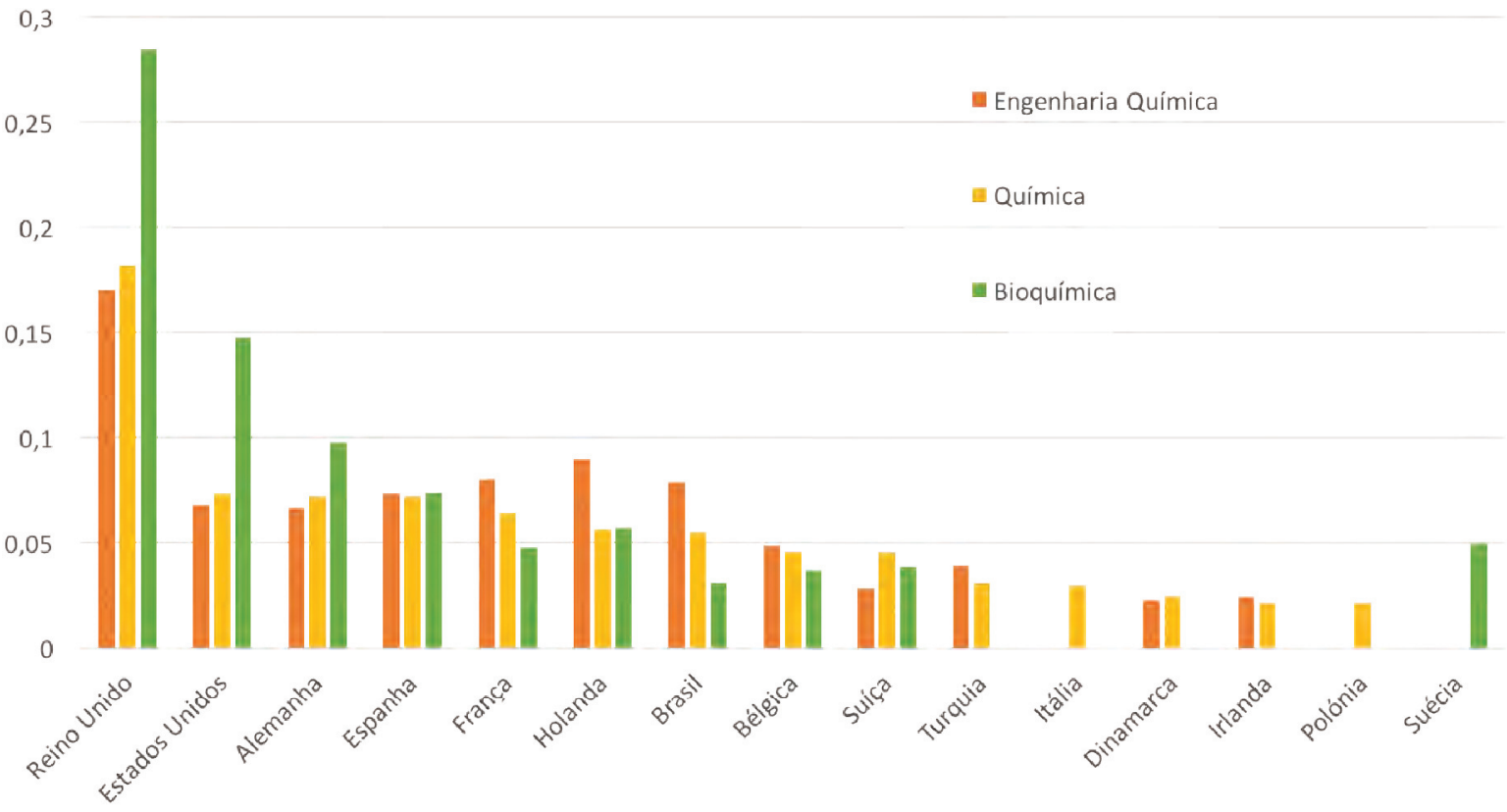

Figura 6 - Distribuição, em termos de fração, dos residentes no estrangeiro por país, dos utilizadores formados em Química e áreas afins. 
mais jovens, este estudo permite ter uma primeira imagem do panorama profissional do químico português. Os dados agora obtidos quando comparados com os resultados do inquérito direto permitirão refinar as conclusões, nomeadamente através da inclusão de dados não disponíveis na rede social.

\section{Metodologias}

Os dados utilizados no presente estudo foram obtidos com a ferramenta "find Alumni" da rede social de negócios LinkedIn [3]. A pesquisa efetuada teve como alvo os profissionais formados em Química, em Engenharia Química e em Bioquímica pelas universidades portuguesas (Algarve, Aveiro, Beira Interior, Coimbra, Évora, Lisboa, Madeira, Minho, Nova de Lisboa, Porto, Trás-os-Montes e Alto Douro). Obteve-se um conjunto com cerca de 6350 utilizadores registados (sem constrangimentos de datas para obtenção da formação ou execução da profissão). A pesquisa foi restrita aos utilizadores que declararam ter pelo menos um dos seguintes graus académicos: Licenciado, Mestre ou Doutor. Dadas as limitações da ferramenta de pesquisa utilizada, um utilizador com graus académicos em duas áreas científicas distintas pode ter sido contabilizado duas vezes. A imagem obtida reporta-se a março de 2016.

\section{Agradecimentos}

Os autores agradecem ao Prof. Doutor Adelino Galvão as discussões úteis sobre o trabalho bem como a ajuda na estruturação do artigo.

\section{Referências}

[1] P.R. Claro, L. Brito, Química - Boletim da SPQ 110 (2008) 45-47.

[2] Inquérito: A Química em Portugal. (2016, 2 de maio). Sítio na rede: http://www.spq.pt/news/540 e www.inquerito.spq.pt

[3] LinkedIn - About. LinkedIn Corporation. (2016, 4 de janeiro). Sítio na rede https://press.linkedin.com/about-linkedin

\section{Atualidades Científicas}

\section{Esferas de carbono com boa capacidade de adsorção de $\mathrm{CO}_{2}$}

Esferas ocas de carbono (HCSs - hollow carbon spheres), também denominadas como cápsulas de carbono, são partículas estruturadas de carbono com tamanhos que podem variar da ordem dos milímetros até à escala nanométrica. Estes materiais têm atraído particular atenção devido às suas propriedades únicas, tais como capacidade de encapsulação, permeabilidade controlável, elevadas razões área superficial/volume e excelente estabilidade química e térmica. Possuem potenciais aplicações em catálise, conversão e armazenamento de energia e adsorção. No entanto, a síntese eficaz de HCSs uniformes, com elevada área superficial e elevada porosidade, permanece um desafio.

Recentemente, uma equipa de investigadores chineses, liderada por Shiyang Bai, da Universidade de Tecnologia de Pequim, desenvolveu um método para preparar HCSs com elevada área superficial e elevada microporosidade. A equipa usou esferas de sílica como modelo, resorcinol (R) e formaldeído (F) como precursores da formação do revestimento de carbono das esferas e tetraetilortosilicato (TEOS) para obtenção dos microporos. As esferas revestidas foram carbonizadas sob uma atmosfera de $\mathrm{N}_{2}$ a $350-600{ }^{\circ} \mathrm{C}$ e, na etapa final, os componentes de sílica foram removidos usando uma solução aquosa de HF. As esferas obtidas mostraram boa aptidão para adsorção de $\mathrm{CO}_{2}$, em particular quando comparada com a das esferas obtidas sem a presença de TEOS. Segundo os autores, estas esferas têm potencial utilização na separação de gases como o $\mathrm{CO}_{2}$ e $\mathrm{N}_{2}$.

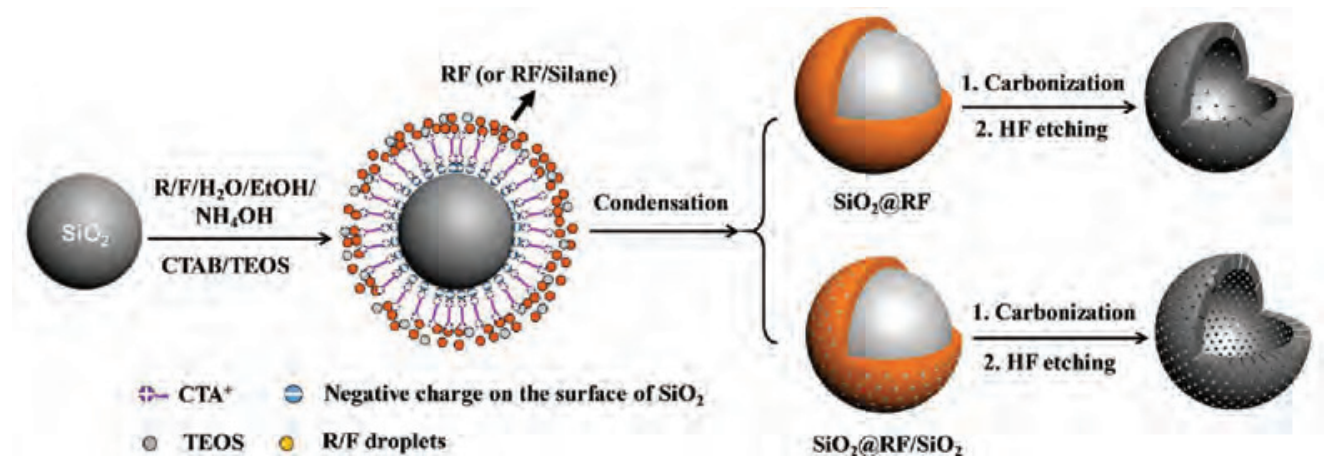

\section{Fontes:}

More Micropores for Better $\mathrm{CO}_{2}$ Absorption, http://www.chemistryviews.org/details/news/10455421/More_Micropores_for_Better_CO2_Absorption.html (Acedido em 03/02/2017)

Xuena Li, Shiyang Bai, Zhengjian Zhu, Jihong Sun, Xiaoqi Jin, Xia Wu, Jian Liu. Hollow carbon spheres with abundant micropores for enhanced $\mathbf{C O}_{2}$ adsorption. Langmuir 33 (2017) 1248-1255. DOI: 10.1021/acs. langmuir.6b04131

Paulo Mendes (pjgm@uevora.pt) 